\title{
Robotic Boreblending: The Future of In-Situ Gas Turbine Repair
}

\author{
David Alatorre, Student Member, IEEE, Bilal Nasser, Amir Rabani, Adam Nagy-Sochacki, \\ Xin Dong, Dragos Axinte*, James Kell
}

\begin{abstract}
Automation of inspection and repair tasks on complex installations is gaining attention from industries with high-value assets such as aerospace, nuclear and marine. This paper reports on a five degrees of freedom robotic system capable of performing accurate and repeatable repair procedures through a narrow inspection port, which minimizes the cost and downtime associated with unscheduled maintenance. Careful study of the target working volume and repair process informed the design of a robotic probe capable of replicating the operation. Kinematic analysis of the robot's flexible, prismatic and rotary joints was used to define accurate machining paths in 3D space, and the results were verified using an optical motion capture system (accuracy of $0.25 \mathrm{~mm}$ ). After comprehensive verifications of the constitutive elements, the robotic system was successfully demonstrated for repair of a high-pressure compressor aerofoil in a gas turbine. The results not only proves the ability of the system to address such difficult repair scenarios but also highlights a domain of opportunities in developing specialist robotics for repair of high-value assets, which is a subject to growing global demand.
\end{abstract}

\section{INTRODUCTION}

In-situ maintenance technicians currently employ a complex customised set of tooling for successive tasks, from visualisation and measurement to surface preparation and material removal. The variety of interventions that need to be performed often results in a significant volume of customized tooling. These operations are currently performed manualy, and therefore lack repeatability and precision. Hence, it follows that a robotic solution for such inspection and repair tasks would be a breakthrough for high-value asset industries with worldwide operations (e.g. aerospace and energy). A robotic approach would offer the possibility for some level of automation of these interventions, eliminating the human errors during manipulation of tools.

The use of robots to automate tasks in inaccessible or inhospitable environments is growing year on year. In [1], Dong et al. demonstrate the use of a long hyper-redundant continuum robot to address repair tasks on the intermediate pressure compression stages of a gas turbine. In [2] and [3], the authors demonstrate the use of a track-based robot to address in-situ a number of processes on hydropower equipment, from post-weld heat treatment to grinding. Robotic instruments are also being deployed for applications such as minimally invasive surgery [4]-[6], where precision, repeatability and smoothness are essential for success.

The application considered here is the realization of a stress relief on a gas turbine compressor blade via a successive set of tasks: navigation into the compressor via

*Corresponding author. Tel.: +44 1159514117

E-mail address: Dragos.Axinte@nottingham.ac.uk a borescope inspection port, positioning of sensors in the required position for measurement of defects, analysis of measurement data, and machining passes to remove the required material. Currently, in-situ boreblending is carried out using manual tools and instruments: a flexible or rigid boroscope such as the ones described in [7] is used to identify the affected area; borescopes with structured light face measurement [8] or stereo vision point cloud [9] capabilities are used to measure the dimensions of the defect; a slender, belt-driven grinding tool with an articulated tip (Figure 1) is used to machine a smooth scallop over the defect. Multiple measurements are taken throughout the procedure to ensure that the stress relief reaches the shape required by the aerospace safety standards, meaning that this procedure can take as much as 3 hours to complete.

This paper presents a novel robotic system which combines continuum type joints [11] with rotary and prismatic stages to replicate the movement of a manual tool, navigate inside a gas turbine and deliver measurement instruments and a grinding end effector to a damaged compressor blade. The available volume within the target environment is analysed in Section II and used to inform the design described in Section III. A kinematic model of the tendon-driven flexible joints is derived and validated experimentally in Section IV. Finally the operation of the robot is demonstrated on a gas turbine in Section V.

\section{DEGREE OF FREEDOM STUDY}

To assess the degree of freedom (DoF) required to perform an automated blending operation, the motion of current manual tools was studied and a computer model of an agreed

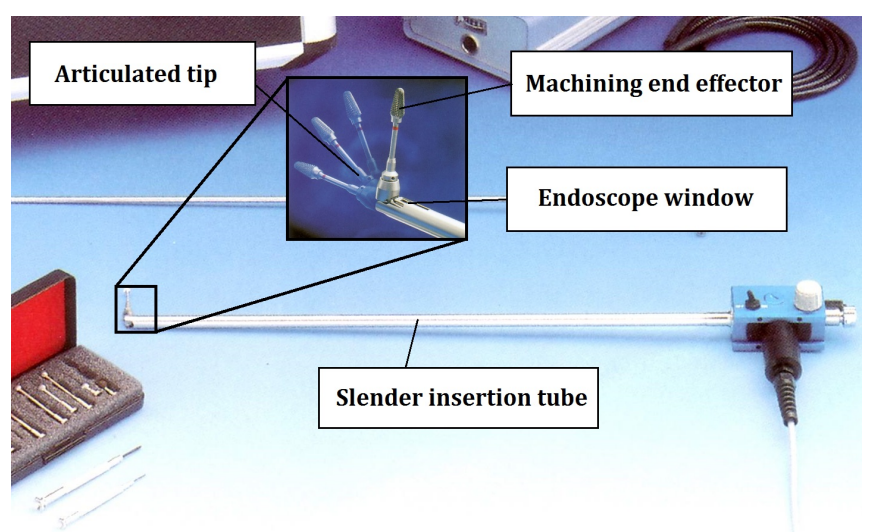

Fig. 1. Conventional boreblending tool for gas turbines (Richard Wolf blending scope [10]) 
set of stages within a gas turbine compressor was evaluated as an example environment. The stage of compression was chosen as a worst case scenario with reduced space for manoeuvring.

Manipulation of existing manual blending tools from outside the casing provides 6 degrees of freedom (Figure $2)$. That being said, some of these allowable motions $\left(X_{0}\right.$, $\left.Y_{0}, A_{0}, B_{0}\right)$ are substantially restricted due to collisions with the environment, as discussed later. The articulation of the tip represents an additional DoF. The pivot joint at the end of the scope is set to $90^{\circ}$ after insertion but, due to the nature of the tool drive shaft, cannot be articulated during operation. The length of the tool and the shape of the cutter are also variable and are therefore relevant to the required DoF for completing abrasion of defects on the compressor blades.

In addition to the movements of the tool there is one degree of freedom associated with the environment, and this is the rotation of the rotor. This DoF can be controlled using a turning tool, a powerful stepper motor that is connected to the turbine gearbox. Because of the location of the turning tool, the backlash and friction of this DoF make it difficult to control the rotor with accuracy and repeatability. It is therefore assumed that once the rotor has been moved to an appropriate position it will not be moved again during operation.

Considering a simplified system (i.e. a blade oriented and fixtured as in a machine tool) an approximation of a perpendicular blend can be achieved with only two degrees of freedom. Setting a plane approximately sectioning the target site $\left(X_{0}-Z_{0}\right.$ plane) allows movement of a tool in $X_{0}$ and $Z_{0}$ to produce a cut (Figure 3 ). The movement in $X_{0}$ could also be approximated by rotation in $C_{0}$, at the expense of squareness of the cut. However, due to the curvature of

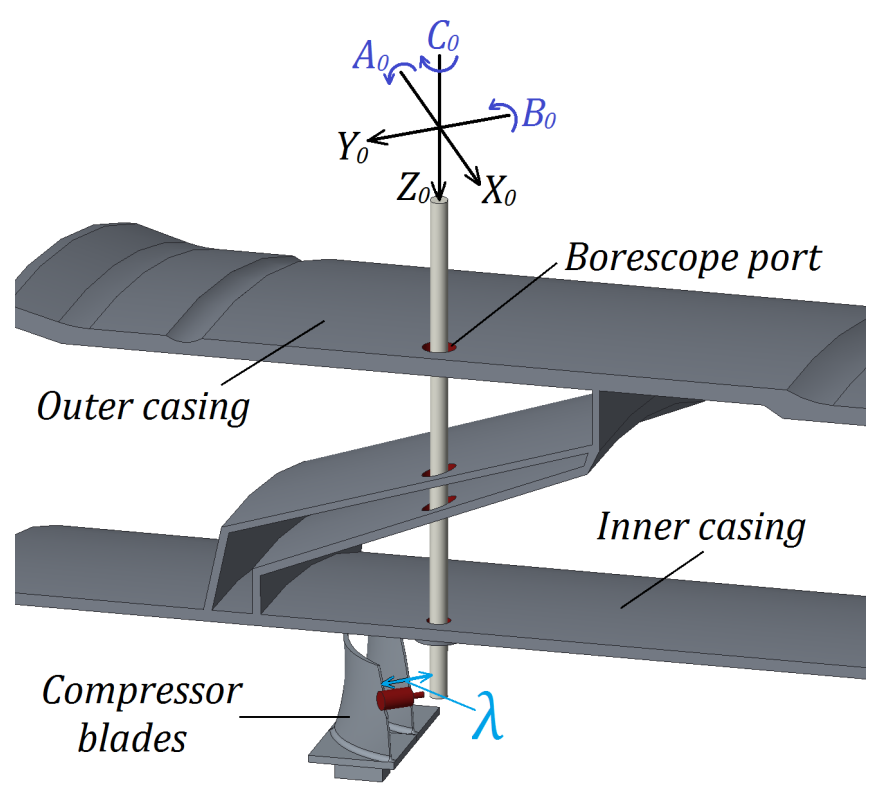

Fig. 2. Cross section of gas turbine borescope port showing degrees of freedom of manual probe (simplified as a cylindrical rod) and rotor blade positions

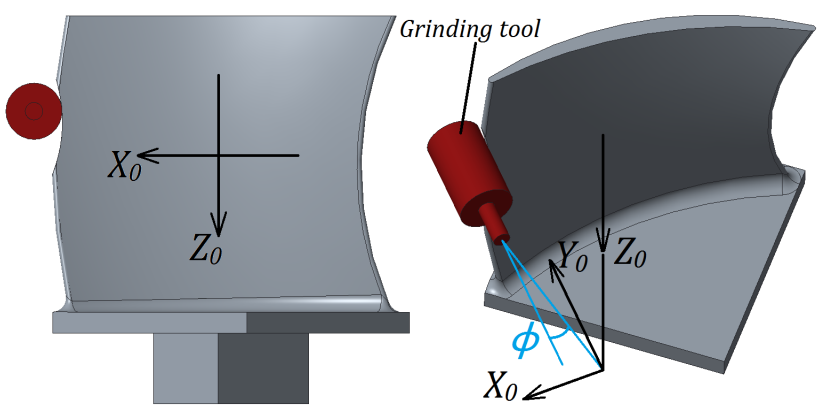

Fig. 3. Simplified system with compressor rotor blade fixed relative to machine tool. $Z_{0}$ marks the axis of the borescope port

compressor blades and the varying angle of attack of the tool ( $\phi$ in Figure 3), some independent articulation in $C_{0}$ is desirable to maintain perpendicularity in this axis. Finally, to maintain perpendicularity to the aerofoil curvature running vertically, some deliberate motion in $A_{0}$ is also desirable. Hence, the required DoFs for full perpendicularity are $X_{0}$, $Z_{0}, A_{0}$ and $C_{0}$.

Access into the compressor is through a series of concentric holes as seen in Figure 2, the first and last of which limit the motion of the tool. Current hand-held boreblending tools, for example, have a maximum linear movement in $X_{0}$ or $Y_{0}$ of $1.2 \mathrm{~mm}$ and a maximum rotation in $A_{0}$ and $B_{0}$ of $2.22^{\circ}$. This shows that even in a scenario where the robotic boreblending tool is as slender as existing tools, translation and rotation in these DoFs is of limited usefulness. Hence, the required degrees of freedom must come from translation in $Z_{0}$, rotation in $C_{0}$ and strategic articulation near the tip. A single DoF of articulation in $A_{0}$ could achieve blends of the required shape but sacrifice perpendicularity of the cut to the blade plane. Two or more orthogonal articulation DoFs at the tip could theoretically achieve the same shape and perpendicularity as a manual tool including accounting for $\phi$. A multiple articulation approach has the added benefit of being able to use the entire diameter of access space.

The distance between the axis of the borescope holes and the edges of the adjacent compressor blades $\lambda$ depends on the position of the rotor, the angles of the nearby stator blade surfaces and the dimensions of the blades themselves. For the stage under consideration this is between $21.5 \mathrm{~mm}$ and 26.7 $\mathrm{mm}$ when the rotor edges are positioned half-way between stator blades (Figure 2). This defines the space available for articulation of the tip and end effector.

Based on this analysis of the required degree of freedom and dimensions, the robotic system was developed and is presented in the section that follows.

\section{SYSTEM ARCHITECTURE}

The design solution that was chosen consists of a hybrid robotic system that makes use of linear and rotary actuators for motion in $Z_{0}$ and $C_{0}$, as well as three tendon-driven flexible joints that articulate the tip within the gas turbine in $A_{0}$ and to some extent in $B_{0}$ and $C_{0}$ (Figure 4). 

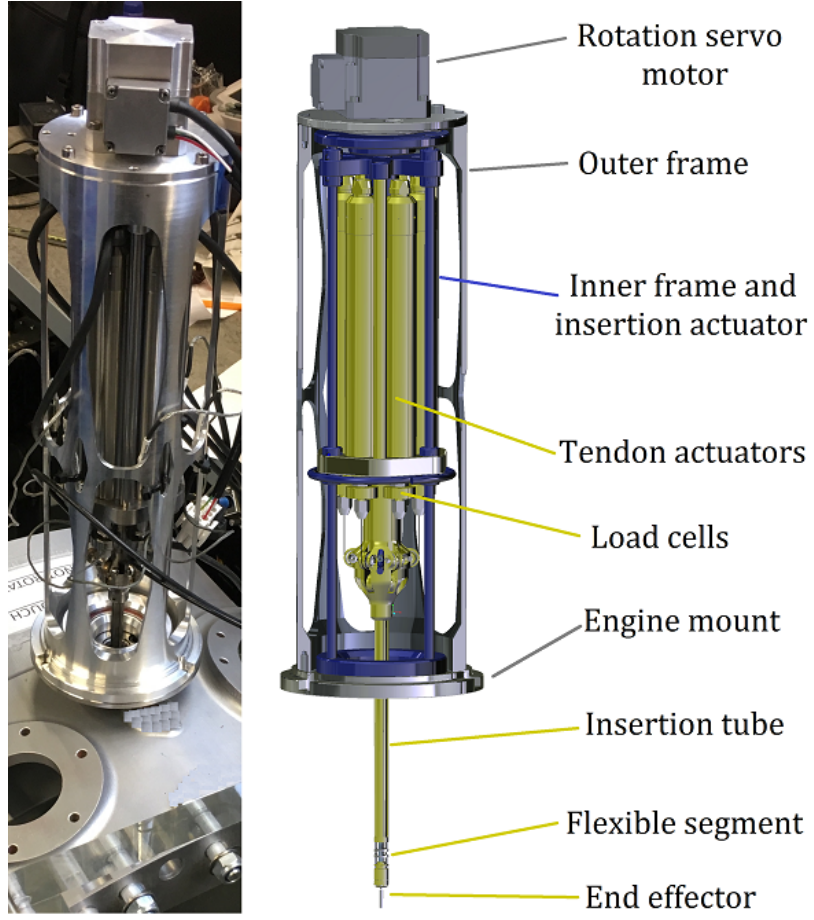

Fig. 4. Mechanical architecture of robotic boreblending robot

A cylindrical outer frame (diameter $100 \mathrm{~mm}$, length 340 $\mathrm{mm}$ ) houses the actuators and sensors that move the $8.5 \mathrm{~mm}$ insertion tube and grinding end effector. This frame is rigidly attached to the outer casing during operation. An FHA-8C-50 rotary servo motor (Harmonic Drive) mounted to the outer frame turns an inner frame (blue in Figure 4) about the axis $C_{0}$ of the borescope port. The inner frame consists of three linear rails and an L-220.50SG linear actuator (P.I.) that slides the insertion tube and relevant actuators (yellow in Figure 4) along inspection port axis $Z_{0}$. Six L-220.20SG linear actuators (P.I.) are used to drive the stainless steel tendons that flex the actuated tip and tilt the end effector, and six LMC201 load cells (Omega Engineering) monitor the cable tensions.

The flexible segments make use of hyper-elastic NiTi backbones that constrain the bend in particular directions, with the added advantage of providing a central umbilical passage for driving of the rotary tool. Due to the high forces required to achieve a 90 degree bend, the articulation in $A_{0}$ was split into two independently controllable flexible joints, each capable of bend angles of $50^{\circ}$. A smaller orthogonal flexible joint was placed between the other two, allowing for $22.5^{\circ}$ of bend (in $C_{0}$ and/or $B_{0}$ depending on inclination) in each direction to achieve perpendicularity at the edge of the blade.

The $8.5 \mathrm{~mm}$ diameter insertion tube also houses the six tendons, a $3 \mathrm{~mm}$ air conduit and a $4 \mathrm{~mm} \mathrm{M}-\mathrm{V}-\mathrm{iQ}$ Video Borescope stereo camera (GEIT) measurement system.

The end effector consists of miniature air driven spindle that has been incorporated in the last stage of the flexible segment. This miniature spindle can hold friction grip tools

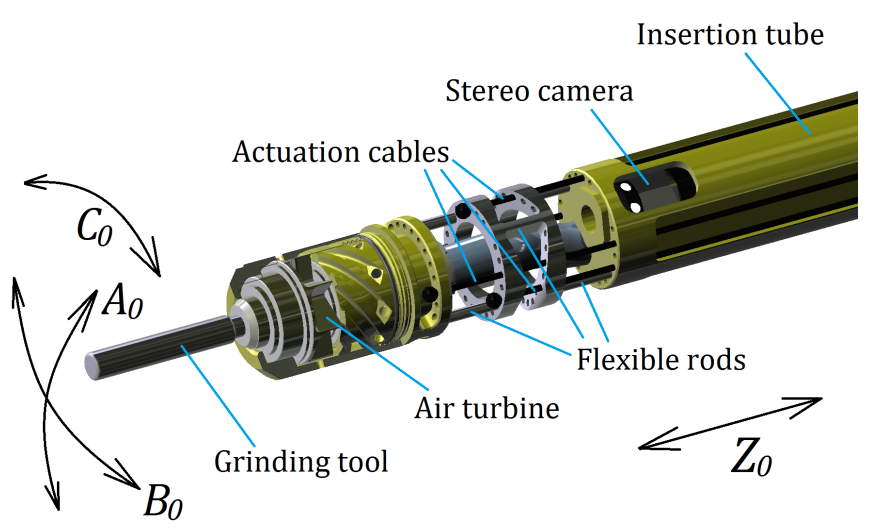

Fig. 5. Detail of measurement probe, flexible articulation and end effector

of $1.6 \mathrm{~mm}$ shaft diameter and achieve speeds of over 250,000 RPM. A $1.8 \mathrm{~mm}$ diameter cylindrical electroplated diamond grinding tool (118 $\mu \mathrm{m}$ grit) was used.

The robot is controlled using a EMP373 (ACME) ruggedised PC that interfaces with the following modules (Figure 6):

- A turning tool controller (Olympus) that provides positioning of the compressor blade under repair

- Two C-843 (P.I.) precision linear actuator controllers that drive robot's seven linear actuators

- A Gold DC Whistle (ELMO Motion Control) servo drive controller that drives the robot's $C_{0}$ rotation stage

- A USB microprocessor (ATMEL) that controls a pneumatic valve that drives the robot's air spindle tool

- A M V-iQ stereo vision measurement system (GE) that uses optical metrology to produce a point cloud of the area of interest

- An iNET-400 data acquisition system (Omega Engineering) that monitors the cable tensions for the flexible joints

\section{KINEMATIC MODEL}

The kinematics is divided into forward and inverse kinematics: the former adapts the Denavit-Hartenberg (D-H) convention mechanism to find the angular and displacement values, and the latter uses the pseudo-inverse Jacobean

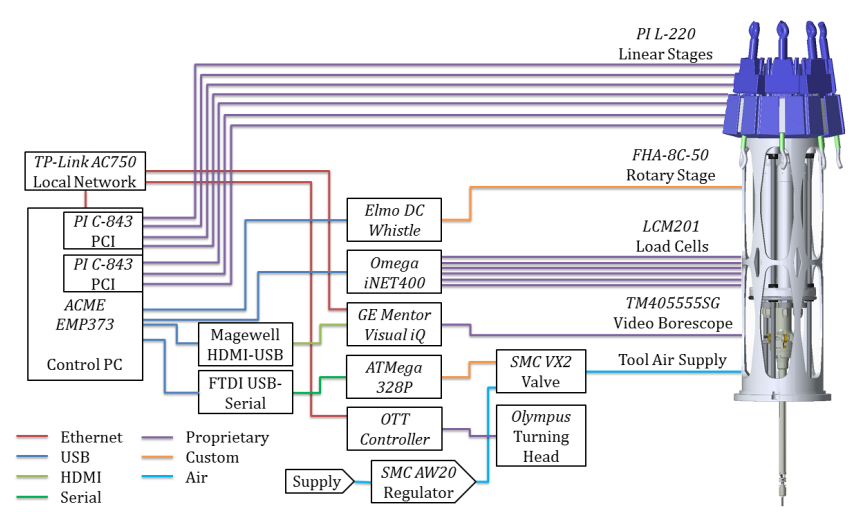

Fig. 6. Hardware schematic for boreblending robot 
functions method to find the optimal solutions for the rigid and flexible (non-linear) joints values to reach the end-effector target with sufficient accuracy.
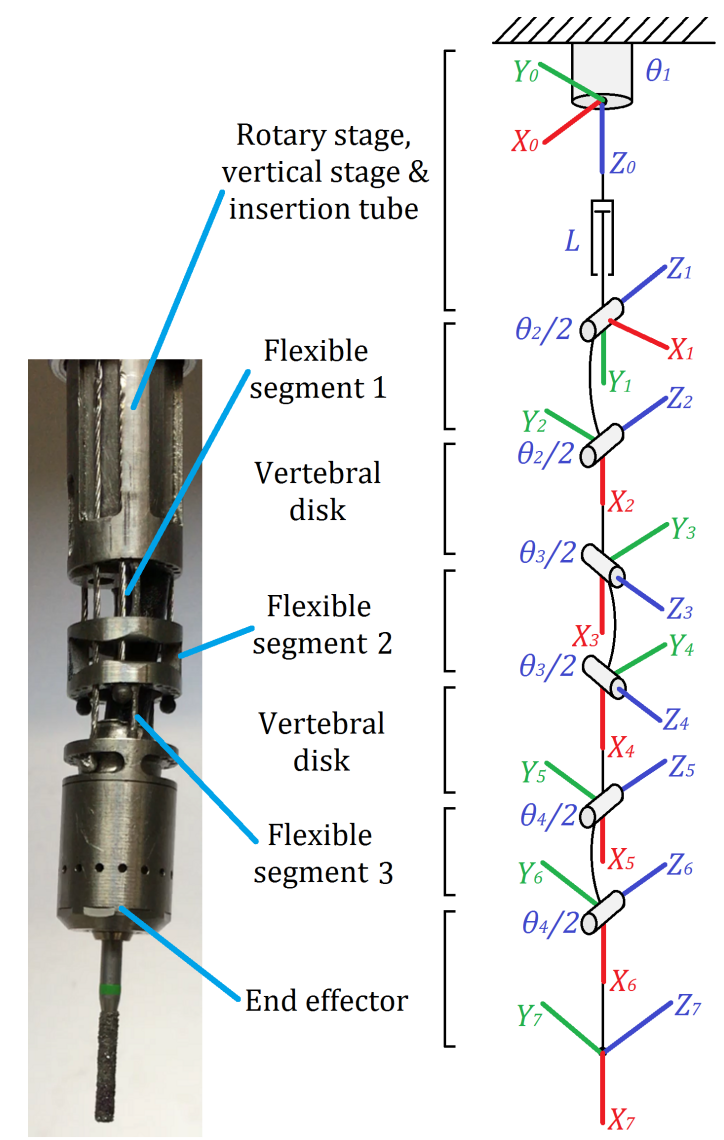

Fig. 7. Denavit-Hartenberg convention diagram for remote boreblending robot

It is assumed that the backbone bends into a circular arc when loaded. Finite element analysis (FEA) of the non-linear hyper-elastic backbone rods suggests that in reality the backbone assumes a tighter bend closer to the ends than in the middle but, due to the dimensions of the components, the assumption only results in an error of $0.16^{\circ}$ or $56 \mu \mathrm{m}$ at the end effector which is within the acceptable tolerance of the blend. Each backbone is described as two revolving joints in the D-H matrix, linked by a rod of varying length. It can be shown that the straight line $d$ between two points of an arc of length $a$ at angle $\theta$ is given by:

$$
d=\sqrt{\frac{2 a^{2}(1-\cos (\theta))}{\theta^{2}}}
$$

with the special case of $d=a$ when $\theta=0$. The two revolving joints either side of each flexible rod share the angle $\theta$ as shown in Figure 7. The D-H parameters of the robot are listed in Table I.

where $a_{1} \ldots a_{7}$ are the lengths of the respective sections and $L$ is the travel of the insertion linear actuator along $Z_{0}$. The robot's forward transformation matrix $T$ can be calculated using

$$
T={ }^{0} T_{1} *{ }^{1} T_{2} *{ }^{2} T_{3} *^{3} T_{4} *{ }^{4} T_{5} *^{5} T_{6} *{ }^{6} T_{7}
$$

TABLE I

HYBRID ROBOT DENAVIT-HARTENBERG CONVENTION PARAMETERS.

\begin{tabular}{|c|c|c|c|c|}
\hline Joint & $\boldsymbol{\theta}$ & $\boldsymbol{\alpha}\left(^{\circ}\right)$ & $\boldsymbol{d}$ & $\boldsymbol{r}$ \\
\hline 1 & $\theta_{1}$ & 90 & $a_{1}+L$ & 0 \\
\hline 2 & $\frac{\theta_{2}}{2}$ & 0 & 0 & $\sqrt{\frac{2 a_{2}^{2}\left(1-\cos \left(\theta_{2}\right)\right)}{\theta_{2}^{2}}}$ \\
\hline 3 & $\frac{\theta_{2}}{2}$ & 90 & 0 & $a_{3}$ \\
\hline 4 & $\frac{\theta_{3}}{2}$ & 0 & 0 & $\sqrt{\frac{2 a_{4}^{2}\left(1-\cos \left(\theta_{3}\right)\right)}{\theta_{3}^{2}}}$ \\
\hline 5 & $\frac{\theta_{3}}{2}$ & -90 & 0 & $a_{5}$ \\
\hline 6 & $\frac{\theta_{4}}{2}$ & 0 & 0 & $\sqrt{\frac{2 a_{6}^{2}\left(1-\cos \left(\theta_{4}\right)\right)}{\theta_{4}^{2}}}$ \\
\hline 7 & $\frac{\theta_{4}}{2}$ & 0 & 0 & $a_{7}$ \\
\hline
\end{tabular}

where, using $c \theta$ and $s \theta$ to signify $\cos (\theta)$ and $\sin (\theta)$,

$$
\begin{gathered}
{ }^{0} T_{1}=\left[\begin{array}{cccc}
c \theta_{1} & 0 & s \theta_{1} & 0 \\
s \theta_{1} & 0 & -c \theta_{1} & 0 \\
0 & 1 & 0 & a 1+L \\
0 & 0 & 0 & 1
\end{array}\right] \\
{ }^{1} T_{2}=\left[\begin{array}{ccccc}
c \frac{\theta_{2}}{2} & -s \frac{\theta_{2}}{2} & 0 & c \frac{\theta_{2}}{2} \sqrt{\frac{2 a_{2}^{2}\left(1-c \theta_{2}\right)}{\theta_{2}^{2}}} \\
s \frac{\theta_{2}}{2} & c \frac{\theta_{2}}{2} & 0 & s \frac{\theta_{2}}{2} \sqrt{\frac{2 a_{2}^{2}\left(1-c \theta_{2}\right)}{\theta_{2}^{2}}} \\
0 & 0 & 1 & 0 \\
0 & 0 & 0 & 1
\end{array}\right] \\
{ }^{6} T_{7}=\left[\begin{array}{cccc}
c \frac{\theta_{4}}{2} & -s \frac{\theta_{4}}{2} & 0 & c \frac{\theta_{4}}{2} a_{7} \\
s \frac{\theta_{4}}{2} & c \frac{\theta_{4}}{2} & 0 & s \frac{\theta_{4}}{2} a_{7} \\
0 & 0 & 1 & 0 \\
0 & 0 & 0 & 1
\end{array}\right]
\end{gathered}
$$

The cable displacement calculations assume a straight line between guide hole centres in the bent configuration.

The inverse kinematics uses the Jacobean pseudo-inverse method [12] to find the optimal solution for each joint in multiple iterations. It is assumed that the intermediate flexible joint angle (for perpendicularity) is defined based on the geometry at the start of the process and remains constant for a given blend. The iterative procedure is as follows:

1) The initial iteration counter is defined as $j=0$

2) The robot's degrees of freedom are annotated as $\mathfrak{q}=$ $\left[\mathfrak{q}_{1}, \mathfrak{q}_{2}, \ldots, \mathfrak{q}_{n}\right]^{\top}$, where $n$ is the total number of DoF

3) To avoid local minima there are four initial guesses defined as $\mathfrak{q}^{(0)} \rightarrow\left\{{ }_{1} \mathfrak{q}^{(0)}{ }_{2} \mathfrak{q}^{(0)}{ }_{3} \mathfrak{q}^{(0)}{ }_{4} \mathfrak{q}^{(0)}\right\}$. These initial guesses were defined based on the geometry and reach of the robot:

$$
\left\{\begin{array}{lll}
\theta_{2}=45, & \theta_{4}=45 & \text { if } Y \geq 20 \\
\theta_{2}=22.5, & \theta_{4}=22.5 & \text { if } 0 \leq Y<20 \\
\theta_{2}=-22.5, & \theta_{4}=-22.5 & \text { if }-20<Y<0 \\
\theta_{2}=-45, & \theta_{4}=-45 & \text { if } Y \leq-20
\end{array}\right.
$$

4) To calculate $\mathfrak{q}^{(j+1)}$, the remainder can be found using:

$$
\delta \mathfrak{w}\left(\mathfrak{q}^{(j)}\right)=J\left(\mathfrak{q}^{(j)}\right) \delta \mathfrak{q}^{(j)}=\mathfrak{w}\left(\mathfrak{q}^{(0)}\right)-\mathfrak{w}\left(\mathfrak{q}^{(j)}\right)
$$


and $\mathfrak{w}$, the configuration vector, can be defined as:

$$
\mathfrak{w}\left(\mathfrak{q}^{(j)}\right)=\left[\frac{\mathfrak{p}_{i}}{\mathfrak{a}_{i} * e^{\frac{\mathfrak{q} n}{\pi}}}\right]
$$

where $\mathfrak{p}_{i}=[T(1,4), T(2,4), T(3,4)]^{\top}$ (from 2) and $\mathfrak{a}_{i}=[T(1,3), T(2,2), T(3,2)]^{\top}[12]$

5) The initial $4 \times 6$ Jacobean matrix used to calculate the joint values can be derived by differentiating the $\mathfrak{w}\left(\mathfrak{q}^{(j)}\right)$, hence:

$$
J\left(\mathfrak{q}^{(j)}\right)=\frac{\delta \mathfrak{w}\left(\mathfrak{q}^{(j)}\right)}{\delta \mathfrak{q}^{(j)}}
$$

Solving $J\left(\mathfrak{q}^{(j)}\right)$ is a matter of using the pseudo-inverse method to calculate $J^{+}\left(\mathfrak{q}^{(j)}\right)$

6) $\mathfrak{q}^{(j+1)}$ is then calculated using:

$$
\mathfrak{q}^{(j+1)}=\mathfrak{q}^{(j)}+J^{+}\left(\mathfrak{q}^{(j)}\right) \delta \mathfrak{w}\left(\mathfrak{q}^{(j)}\right)
$$

7) The last step to find a suitable solution is to set a tolerance value $\epsilon$ close to zero and checking whether the new joint coordinate values are within $\epsilon$ of the target:

$$
\begin{cases}\text { Solution is found } & \text { if } \mathfrak{q}^{(j+1)}-\mathfrak{q}^{(j)}<\epsilon \\ j=j+1, \text { repeat from 4) } & \text { otherwise }\end{cases}
$$

The Kinematic model was validated using an optical motion capture system (Vicon Motion Systems Ltd) to track the 3D motion of the robot's end effector. The system consists of four high-resolution Vantage 16 cameras and five retro-reflective passive markers positioned on a surface attached to the end effector of the robot (Figure 8).

The robot was commanded to follow a pre-defined arc with the end effector on the $Y_{0}-Z_{0}$ plane such that, at

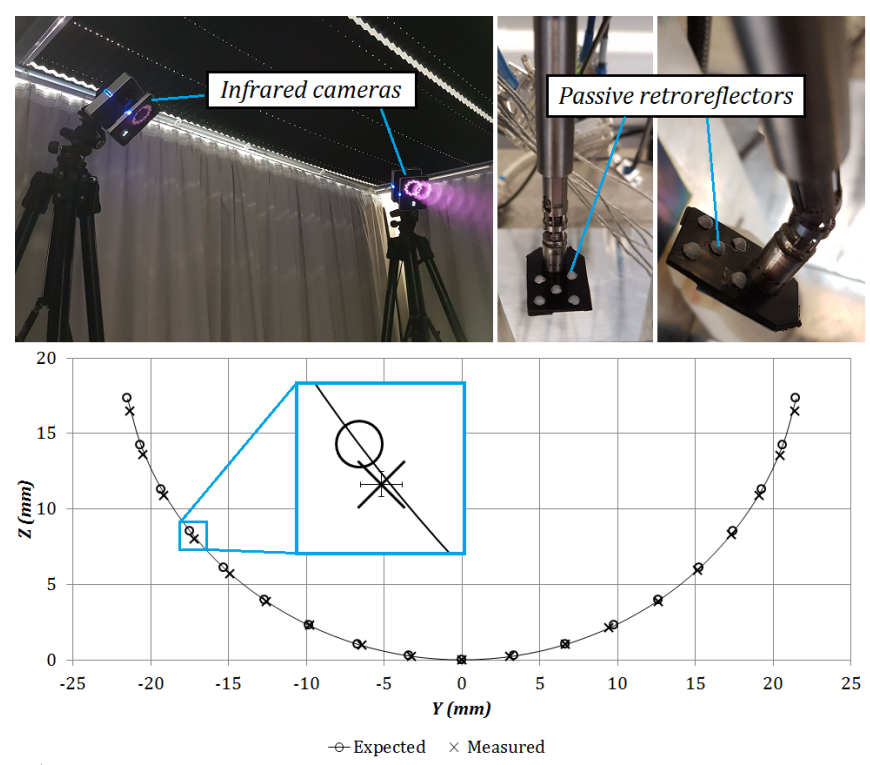

Fig. 8. Kinematics validation experiment setup (top) and position results (bottom) using optical motion capture system. Error bars visible in expanded view show typical st. dev. any point, the unique solution has the distal and proximal flexible segments bent equally. Discrete measurements were taken at 5 degree intervals. The results (Figure 8) show high correlation between the expected angular motion of the robot's individual joints and the actual movement with an average standard deviation of $0.94^{\circ}$ or $0.26 \mathrm{~mm}$ at the tip of the air spindle housing. It is assumed that the errors in position were caused by insufficient or asymmetric pre-tension on the tendons, as well as elasticity of the cables under load. Despite these errors, the results confirm the validity of the kinematic model.

\section{SYSTEM DEMONSTRATION}

The system was demonstrated first using a representative test rig in a lab environment and then on an appropriate gas turbine compressor.

To study the repeatability of the system, eleven compressor blades were intentionally damaged on the leading edge with similar defects (1.5 mm wide, $800 \mu \mathrm{m}$ deep) on a mock-up compressor stage (Figure 9). The robot was inserted into the borescope port and the defects were scanned using the embedded stereo measurement camera to identify the target area from the point cloud. Each measurement was used to automatically generate a stress relief machining path using aerospace standard specifications of aspect ratio and shape. The start point of the machining path was defined based on the height of the measurement camera above the tool bending plane. To prevent the air turbine tool from stalling upon contacting the blade, the machining operation was broken up into thirty passes with a maximum depth of cut of 30.7 $\mu \mathrm{m}$.

Once the blends were completed, each blade was scanned using a structured light 3D scanner (Artec Spider) with
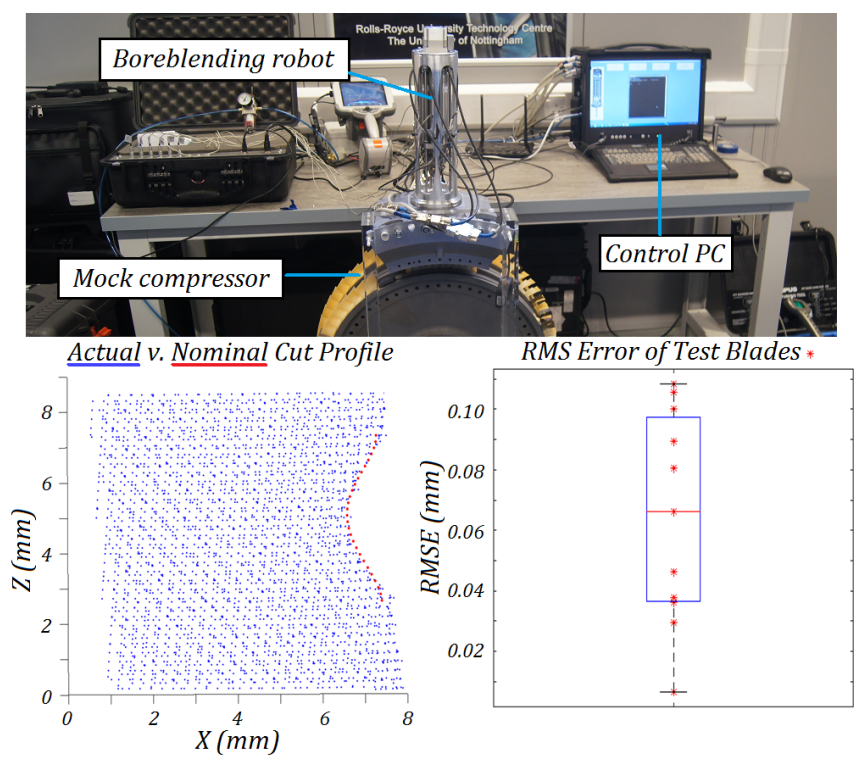

Fig. 9. Experimental setup (top) and results (bottom) of repeatability study on compressor test rig. Bottom left plot shows experimental point cloud data of blade compared to expected tool path, bottom right plot shows error for each of the blades tested. 


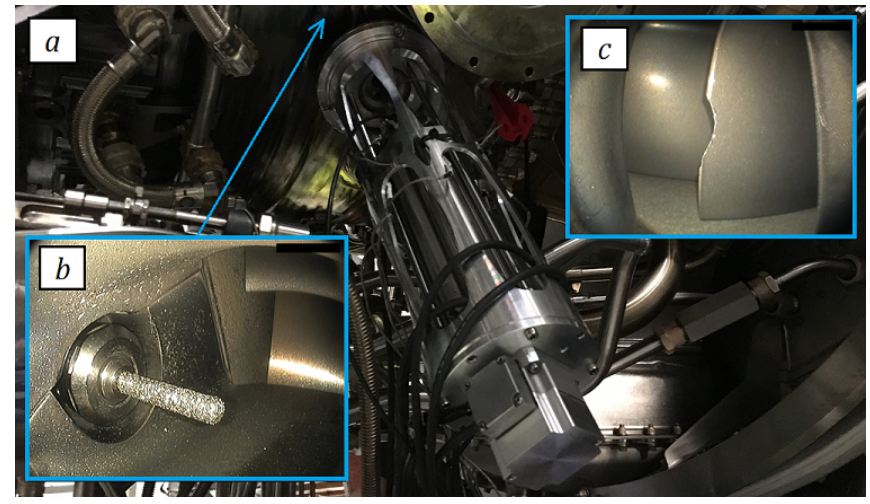

Fig. 10. Boreblending robot mounted on gas turbine inspection port (a), view of robot end effector from inside engine (b) and finished scallop blend (c)

a quoted accuracy of $0.05 \mathrm{~mm}$. The point clouds were compared to nominal simulated machining curves using the iterative closest point registration method. The results show strong correlation between the point clouds and the nominal curves, with a median RMSE of $64 \mu \mathrm{m}$ and a standard deviation between blades of $28 \mu \mathrm{m}$ (Figure 9). Consistent dimensions were observed along all eleven blades, confirming the repeatability of measurement and machining. The average blend duration was 45 minutes, demonstrating a substantial improvement over the manual method.

Finally, the robot's capabilities were demonstrated on a production jet engine (Figure 10) with a previously identified defect. The robot was inserted through the boroscope port and moved to the inspection position; measurements of the defect were made using the built-in probe; and the point cloud was analysed to fit the correct scallop curve. The robot machined the blade over 54 minutes (more passes were used than during the lab tests as the defect was larger) and retracted back out of the borescope port to be removed from the engine. After inspection by a trained professional, the shape, size and finish of the scallop were deemed adequate for aerospace standards, demonstrating the applicability of such robotics endeavours.

While the example scenario chosen for demonstration is fairly specific, the approach is scalable to other repair tasks usually carried out manually through narrow passages, and could be adapted to suit the needs of inaccessible parts of industrial hardware such as turbines and compressors or key parts of hazardous hardware such as nuclear reactors.

\section{CONCLUSIONS}

This paper presents for the first time the hardware architecture of a robotic system for in-situ boreblending on gas turbines.

The degree of freedom of current manual boreblending tools was analysed and the results were used to inform the design of a 5 degree of freedom robotic probe fitted with 3D measurement instrumentation and an air-driven grinding tool for machining. The robot moves using a combination of rotary, prismatic and continuum joints for a novel hybrid architecture well suited for work in confined spaces.

The forward and inverse kinematics of this hybrid design were derived using the Denavit-Hartenberg convention and the Jacobian pseudo-inverse method and tested experimentally to be capable of navigating its flexible joints with $0.26 \mathrm{~mm}$ accuracy.

The system was put to the test by machining a number of stress relief scallops on compressor blades with a shape RMS error of $0.064 \mathrm{~mm}$, and was demonstrated by removing a defect on a gas turbine compressor blade. The demonstration showed the ability of the system to reduce the time taken for the operation and improve the repeatability.

This work presents an approach to tackle automation tasks in confined and challenging environments which is scalable and flexible. The resulting hardware has been shown to provide value to industries with high-value assets and lays the foundations for fully automated in-situ repair.

\section{ACKNOWLEDGMENT}

The authors would like to acknowledge the funding support from the Aerospace Technology Institute for the works presented as a part of the SAMULET II Advanced Repair Technologies project. A special thanks to Rolls-Royce Plc for their help during the engine demonstration.

\section{REFERENCES}

[1] X. Dong, D. Axinte, D. Palmer, S. Cobos, M. Raffles, A. Rabani, and J. Kell, "Development of a slender continuum robotic system for on-wing inspection/repair of gas turbine engines," Robotics and Computer-Integrated Manufacturing, vol. 44, pp. 218-229, 2017.

[2] E. Boudreault, B. Hazel, J. Cote, and S. Godin, "In situ post-weld heat treatment on martensitic stainless steel turbine runners using a robotic induction heating process to control temperature distribution," 27th Iahr Symposium on Hydraulic Machinery and Systems (Iahr 2014), Pts 1-7, vol. 22, 2014.

[3] B. Hazel, J. Cote, Y. Laroche, and P. Mongenot, "A portable, multiprocess, track-based robot for in situ work on hydropower equipment," Journal of Field Robotics, vol. 29, no. 1, pp. 69-101, 2012

[4] A. Degani, H. Choset, A. Wolf, and M. A. Zenati, "Highly articulated robotic probe for minimally invasive surgery," 2006 Ieee International Conference on Robotics and Automation (Icra), Vols 1-10, pp. 4167-4173, 2006

[5] C. He, S. X. Wang, H. Q. Sang, J. H. Li, and L. N. Zhang, "Force sensing of multiple-dof cable-driven instruments for minimally invasive robotic surgery," International Journal of Medical Robotics and Computer Assisted Surgery, vol. 10, no. 3, pp. 314-324, 2014.

[6] J. N. Ding, R. E. Goldman, K. Xu, P. K. Allen, D. L. Fowler, and N. Simaan, "Design and coordination kinematics of an insertable robotic effectors platform for single-port access surgery," Ieee-Asme Transactions on Mechatronics, vol. 18, no. 5, pp. 1612-1624, 2013.

[7] W. J. Lang, "The art of borescope photography," Materials Evaluation, vol. 45 , no. 12 , pp. $1361-1370,1987$

[8] R. J. Valkenburg and A. M. McIvor, "Accurate 3d measurement using a structured light system," Image and Vision Computing, vol. 16, no. 2, pp. 99-110, 1998.

[9] H. Kieu, T. Y. Pan, Z. Y. Wang, M. Le, H. Nguyen, and M. Vo, "Accurate $3 \mathrm{~d}$ shape measurement of multiple separate objects with stereo vision," Measurement Science and Technology, vol. 25, no. 3, 2014.

[10] Richard Wolf GmbH, "Richard wolf blending scope." [Online] Available: http://www.blending-scope.com/ [Accessed: 01-Mar-2018]

[11] X. Dong, M. Raffles, S. C. Guzman, D. Axinte, and J. Kell, "Design and analysis of a family of snake arm robots connected by compliant joints," Mechanism and Machine Theory, vol. 77, pp. 73-91, 2014

[12] C. A. Klein and C. H. Huang, "Review of pseudoinverse control for use with kinematically redundant manipulators," Ieee Transactions on Systems Man and Cybernetics, vol. 13, no. 2, pp. 245-250, 1983. 